\title{
Viabilidade do cultivo do camarão-rosa Farfantepenaeus paulensis (Crustácea, Decapoda) em gaiolas sob diferentes densidades durante o outono no sul do Brasil
}

\author{
Viability of culturing the shrimp Farfantepenaeus paulensis (Crustacea, Decapoda) in cages under different \\ stocking densities during autumn in southern Brazil
}

\author{
Dariano Krummenauer ${ }^{1}$ Wilson Wasielesky Júnior ${ }^{2}$ Ronaldo Oliveira Cavalli ${ }^{2}$ \\ Silvio Peixoto ${ }^{2}$ Paulo Roberto Zogbi²
}

\section{RESUMO}

Neste trabalho, foi estudada a possibilidade de cultivar o camarão-rosa Farfantepenaeus paulensis em gaiolas com diferentes densidades de estocagem durante o outono (abril a junho) no estuário da Lagoa dos Patos, RS. Juvenis com peso médio de 4,95g ( $\pm 1,69 \mathrm{dp}$ foram estocados em gaiolas nas densidades de 20, 40, 80, 100 e $120 \mathrm{ind} / \mathrm{m}^{2}$. Os camarões foram alimentados diariamente ad libitum com pedaços de Corvina (Micropogonias furnieri). As sobrevivências e os pesos foram observados nos tempos 20, 40 e 60 dias de experimento. Durante o período de cultivo a temperatura da água decresceu de 24 para $11^{\circ} \mathrm{C}$ (média $=17,1^{\circ} \mathrm{C}$ ) e a salinidade oscilou entre 3 e 26 (média =17,5). As taxas de sobrevivência nas densidades de 20, 40, 80, 100 e $120 \mathrm{ind} / \mathrm{m}^{2}$ foram $65 \%$, $68 \%, 46 \%, 52 \%$ e $43,3 \%$ respectivamente, sendo significativamente maiores nas densidades de 20 e $40 \mathrm{ind} / \mathrm{m}^{2}$. Após 60 dias, os camaróes estocados em 20 e $40 \mathrm{ind} / \mathrm{m}^{2}$ atingiram peso superior a 8,0g, sendo significativamente maiores $(P<0,05)$ que nos demais tratamentos. A maior biomassa (356g) foi obtida na densidade de $120 \mathrm{ind} / \mathrm{m}^{2}$. Foram produzidos de 250 a $350 \mathrm{~g}$ de camarões com peso médio de 6,5 a 8,0g por $m^{2}$, mesmo em altas densidades de estocagem (40 a $120 \mathrm{ind} / \mathrm{m}^{2}$ ). As taxas de sobrevivência dos camarões mantidos em 20 e $40 \mathrm{ind} / \mathrm{m}^{2}$ podem ser consideradas satisfatórias, contudo, após a temperatura ficar abaixo de $18^{\circ} \mathrm{C}$, as taxas de crescimento foram reduzidas. Os resultados confirmam o efeito negativo da densidade de estocagem sobre o crescimento dos camarões, sendo recomendada a estocagem de 40 a 120 juvenis $/ \mathrm{m}^{2}$. Foi, contudo, comprovada a possibilidade de cultivar $\boldsymbol{F}$. paulensis durante o outono no sul do Brasil.

Palavras-chave: Farfantepenaeus paulensis, gaiolas, densidade de estocagem, cultivo no outono.

\begin{abstract}
In this study, we assessed the feasibility of culturing Farfantepenaeus paulensis in cages at different stocking densities during autumn (from April to June) in the Patos Lagoon estuary. Juveniles with mean weight of $4.95 \mathrm{~g}( \pm 1.69$ SD) were stocked in cages at densities of 20,40, 80, 100 and $120 \mathrm{ind} / \mathrm{m}^{2}$ of bottom. Shrimp were fed daily by-catch items (Micropogonias furnieri) ad libitum. Survival and wet weight were estimated at 20, 40 and 60 days after the beginning of the trial. Water temperature varied from 11 to $24^{\circ} \mathrm{C}\left(\right.$ mean $\left.=17.1^{\circ} \mathrm{C}\right)$, whiles salinity ranged from 3 to $26($ mean $=17.5)$. Survival rates at densities of $20,40,80,100$ and $120 \mathrm{ind} / \mathrm{m}^{2}$ were $65 \%$, $68 \%, 46 \%, 52 \%$ and $43,3 \%$, respectively, being significantly higher at 20 and $40 \mathrm{ind} / \mathrm{m}^{2}$. After 60 days, shrimp stocked at 20 and $40 \mathrm{ind} / \mathrm{m}^{2}$ reached over $8.0 \mathrm{~g}$, which were significantly higher than those reared at 80, 100 and $120 \mathrm{ind} / \mathrm{m}^{2}$. The highest biomass $\left(356 \mathrm{~g} / \mathrm{m}^{2}\right)$ was obtained at 120 shrimp per $m^{2}$. Up to 250 to $350 \mathrm{~g}$ of shrimp with mean weight of 6.5 to $8.0 \mathrm{~g}$ were produced per $m^{2}$ even at the highest stocking densities (40 to $120 \mathrm{ind} / \mathrm{m}^{2}$ ). Survival rates of shrimp reared at 20 and $40 \mathrm{ind} /$ $m^{2}$ may be considered satisfactory, whereas growth rates where negligible after temperature dropped below $18^{\circ} \mathrm{C}$. The present results confirmed the negative effect of stocking density on shrimp growth, being recommended the stocking of at the most 40 to $120 \mathrm{ind} / \mathrm{m}^{2}$. Although the feasibility of culturing $\boldsymbol{F}$. paulensis in cages during autumn in southern Brazil was demonstrated.
\end{abstract}

Key words: Farfantepenaeus paulensis, cages, stocking densities, off-season culture.

\section{INTRODUÇÃO}

O camarão-rosa Farfantepenaeus paulensis distribui-se ao longo da plataforma continental brasileira, a partir de Ilhéus, Bahia, estendendo-se pela

${ }^{1}$ Departamento de Oceanografia, Fundação Universidade Federal de Rio Grande (FURG), 96201-900, Rio Grande, RS, Brasil. E mail: darianok@gmail. com.

${ }^{2}$ Departamento de Oceanografia, FURG, Rio Grande, RS, Brasil. 
plataforma do Uruguai até o litoral nordeste da Argentina (D`INCAO 1991). No estuário da Lagoa dos Patos, RS, é capturado durante o verão e início de outono (D'INCAO, 1991). As safras anuais deste camarão no estuário apresentam grandes flutuações de captura, com uma produção média de aproximadamente 3500 toneladas/ano. Embora em alguns anos a produção pesqueira no estuário atinja valores de até 8.000 toneladas, em outros, a safra é inexistente, provocando desemprego e diminuindo significativamente a renda das pessoas ligadas ao setor (D'INCAO et al., 2002).

Diante desse quadro, o cultivo de camarões marinhos em estruturas alternativas, como gaiolas e cercados, pode permitir que comunidades de pescadores artesanais tenham acesso a uma renda suplementar. Esses tipos de estruturas vêm sendo utilizados para cultivar camarões em varias regiões do mundo, pois apresentam um baixo custo de implantação e manejo, alem de aproveitar a produtividade natural do ambiente. No Brasil, o cultivo em gaiolas do camarão exótico Litopenaeus vannamei foi estudado nas regiões nordeste (PAQUOTTE et al., 1998) e sudeste (LOMBARDI et al., 2001). Entretanto, no extremo sul do Brasil, os estudos de cultivo em gaiolas e cercados na região do estuário da Lagoa dos Patos estão sendo desenvolvidos com a espécie nativa $\boldsymbol{F}$. paulensis (DOMINGOS 2000; JENSEN et al. 2003; BALLESTER et al. 2003).

A densidade de estocagem de camarões é um dos principais fatores que determinam a sobrevivência, o crescimento e, conseqüentemente, a biomassa final produzida em um cultivo (WYBAN \& SWEENEY, 1991). Adensidade de estocagem ideal pode variar em função da espécie, das estratégias de manejo de cultivo ou de parâmetros ambientais (WASIELESKY, 2000). Desta forma, o conhecimento dos níveis máximos de estocagem permite otimizar a produtividade dos sistemas de cultivo.

No sul do Brasil, a temperatura diminui no outono, o que pode afetar as taxas de sobrevivência e crescimento dos camarões cultivados (WASIELESKY, 2000). Entretanto, PEIXOTO et al. (2003) observaram que, durante o cultivo de $\boldsymbol{F}$. paulensis em viveiros, o crescimento não foi afetado por temperaturas relativamente baixas $\left(19^{\circ} \mathrm{C}\right)$ durante o outono no sul do Brasil. Neste contexto, o cultivo de $\boldsymbol{F}$. paulensis, mesmo sob condições de temperatura sub-ótimas, pode ser justificado pela disponibilidade de camarão vivo após o encerramento da temporada de pesca da espécie (final do verão), o que usualmente resulta em melhores preços de mercado.

O presente trabalho teve como objetivo analisar a possibilidade de cultivar $\boldsymbol{F}$. paulensis em gaiolas sob diferentes densidades de estocagem durante o outono (abril a junho) no estuário da Lagoa dos Patos.

\section{MATERIAL E MÉTODOS}

O experimento foi realizado durante o outono de 1997, entre os dias 18 de abril e 18 de junho, totalizando 60 dias. As gaiolas foram colocadas na Enseada do Saco do Justino (32 03' 55" S - 52 ${ }^{\circ} 12^{\prime} 30^{\prime \prime}$ W), localizada na região estuarial da Lagoa dos Patos, Rio Grande, RS, Brasil.

As densidades de estocagens testadas foram $20,40,80,100$ e 120 camarões $/ \mathrm{m}^{2}$ com três repetições por tratamento. O peso médio inicial ( \pm DP) dos camarões foi $4,95 \pm 1,69 \mathrm{~g}$. Foram utilizadas 15 gaiolas armadas com estruturas de ferro (vergalhões de ferro com 3/8“de diâmetro). A área total de fundo de cada gaiola foi de $1,0 \mathrm{~m}^{2}$ e altura de $0,40 \mathrm{~m}$. As gaiolas foram revestidas externamente com rede de poliamida (PA), malha com abertura de 5,0 mm entre nós opostos e entralhada com fio multifilamento (240/12).

A alimentação foi oferecida ad libitum diariamente as $17 \mathrm{~h} 30 \mathrm{~min}$. Foi utilizado como alimento, pedaços de Corvina (Micropogonias furnieri). Sua composição centesimal apresenta 77,62\% (umidade), 18,15\% (proteína bruta), 1,9\% (lipídeos totais), 2,50\% (carboidratos), $0,36 \%$ (cinzas). Os peixes foram eviscerados, triturados e pesados em balança digital (precisão de $\pm 0,01 \mathrm{~g}$ ).

A taxa de arraçoamento foi de $20 \%$ da biomassa no início, sendo reduzida ao longo do experimento até alcançar 5\% da biomassa no final. Diariamente às 08 horas da manhã foi realizado o acompanhamento da temperatura (termômetro de mercúrio $-10 \mathrm{a}+50^{\circ} \mathrm{C}$ ) e salinidade (refratômetro AOScientific Instruments Warnner - Lambert). A cada 20 dias, todos os camarões foram retirados das gaiolas e contados para determinar a taxa de sobrevivência ao longo do cultivo. Aleatoriamente foram selecionados e pesados 20 camarões de cada gaiola. As taxas de crescimento semanal foram calculadas de acordo com a fórmula $(\mathrm{PF}-\mathrm{PI} / \mathrm{T}) \times 7$, onde: $\mathrm{PF}=$ peso final; $\mathrm{PI}=$ peso inicial e $\mathrm{T}=$ intervalo de tempo entre biometrias sucessivas (dias).

O peso final e as taxas de crescimento semanal de cada uma das três repetições foram submetidos à análise de variância (ANOVA) levandose em considerações as premissas necessárias. Não sendo detectadas diferenças significativas $(\mathrm{P}>0,05)$ entre as repetições, as mesmas foram agrupadas. Os resultados de sobrevivência foram transformados pelo arco-seno da raiz quadrada para realização das análises, 
mas somente os valores originais são apresentados. As diferenças nas médias de peso e sobrevivência dos camarões entre os tratamentos foram verificadas por ANOVA, seguida do teste de Tukey quando constatadas diferenças significativas $(\mathrm{p}<0,05)$.

\section{RESULTADOS}

A temperatura média da água diminuiu gradativamente ao longo do período experimental, oscilando entre $24,0^{\circ} \mathrm{C}$ e $11,0^{\circ} \mathrm{C}$ (Figura 1). A salinidade oscilou entre 26 e 3 , apresentando um valor médio de 17,5 .

Ao final do experimento, as taxas médias de sobrevivência nas densidades de 20, 40, 80, 100 e 120 camarões $/ \mathrm{m}^{2}$ foram respectivamente $65 \%, 68 \%, 46 \%$, $52 \%$ e $43,3 \%$. Estes resultados não foram significativamente diferentes $(\mathrm{P}>0,05)$ (Figura 2). $\mathrm{O}$ peso médio final $( \pm \mathrm{dp})$ dos camarões nas densidades de estocagem de 20,40, 80, 100 e 120 camarões $/ \mathrm{m}^{2}$ foi de $8,2( \pm 1,79), 8,12( \pm 1,62), 6,51( \pm 1,61), 6,52( \pm 2,13), 6,87$ $( \pm 2,47)$, respectivamente (Figura 3$)$. Foram detectadas diferenças significativas $(\mathrm{P}<0,05)$ entre os pesos finais dos diferentes tratamentos, sendo os maiores valores observados nas densidades de 20 e 40 camarões $/ \mathrm{m}^{2}$.

As taxas de crescimento semanal para as diferentes densidades de estocagem estão apresentadas na tabela 1 . No período inicial (0 a 20 dias), as taxas foram mais elevadas, sendo observadas diferença significativa entre os diferentes tratamentos. Entretanto, durante o período intermediário (20-40 dias) e final (40-60 dias) as taxas não foram diferentes entre as densidades de estocagem testadas.

\section{DISCUSSÃO}

A temperatura é um dos fatores limitantes que pode alterar as taxa de sobrevivência e crescimento dos camarões, tanto no ambiente natural quanto em sistemas de cultivo (JACKSON \& WANG, 1998; WASIELESKY, 2000). LOWERY (1988) aponta a temperatura como um dos principais fatores que afetam o processo de muda e o crescimento dos crustáceos. LIAO \& CHIEN (1994) indicaram que o camarão Marsupenaeus japonicus tem um ótimo de crescimento entre 25 e $30^{\circ} \mathrm{C}$. Segundo SOARES et al., (2000), a temperatura ideal para o crescimento de pós-larvas de F. paulensis está entre $29^{\circ} \mathrm{C}$ e $32^{\circ} \mathrm{C}$, muito embora OLIVERA et al. (1993) tenham demonstrado o crescimento desta espécie a $15^{\circ} \mathrm{C}$. No presente trabalho, a temperatura média decresceu ao longo do período experimental atingindo valores que afetam o crescimento da espécie. Apesar disso, os valores mínimos atingidos estão dentro da faixa de tolerância da espécie (WASIELESKY, 2000). Apesar das taxas de crescimento, durante os 20 primeiros dias deste trabalho, estarem de acordo com os resultados de WASIELESKY, (2000), o período restante de cultivo apresentou taxa praticamente nula, o que claramente indica o efeito do declínio da temperatura ( $35^{\circ}$ dia), ocorrido entre a segunda e terceira amostragem de camarões.

Além da temperatura, a salinidade também é um importante parâmetro que influencia as respostas funcionais de organismos aquáticos, tais como sobrevivência, metabolismo, crescimento e reprodução (KINNE, 1964). No presente trabalho, a salinidade apresentou uma ampla variação ao longo do experimento (3 a 26), sendo este fenômeno característico do estuário da Lagoa dos Patos. WASIELESKY (2000) encontrou na salinidade de 22,93 o ponto isosmótico para juvenis da mesma espécie. $\mathrm{O}$ mesmo autor verificou que juvenis de $\boldsymbol{F}$. paulensis apresentam potencial para ser cultivado em salinidades entre 5 e 40, sem apresentar mortalidades significativas. O valor médio de salinidade no presente estudo está de acordo com MUEDAS et al. (1997), que indicaram que a faixa ideal para o cultivo deste camarão estaria compreendida entre 15 e 25 . Como a média de salinidade, no presente estudo, esteve dentro da faixa indicada (média de 17,5), acredita-se que este parâmetro não tenha afetado de forma significativa o crescimento e a sobrevivência dos camarões.

Embora o estuário da Lagoa dos Patos tenha uma alta produtividade natural, as taxas de sobrevivência podem ter sido afetadas pelo tipo de alimento fornecido aos camarões. Em estudo recente, SANTOS (2003) verificou que $\boldsymbol{F}$. paulensis, alimentado somente com peixes, apresentou alta mortalidade a partir da segunda semana de cultivo, de forma similar,ao que é relatado no presente trabalho. Foi observado um pico de mortalidade a partir da terceira semana de cultivo. Este autor cita ainda que este fato pode estar associado a uma deficiência e/ou ausência de composto(s) nutricional (is) imprescindível (is) aos processos vitais. Da mesma forma, DOMINGOS (2000) observou mortalidades significativas de juvenis de $F$. paulensis cultivados em gaiolas no ambiente estuarino e alimentados unicamente com músculo de corvina salgado (M. furnieri). Em condições de temperatura e salinidade semelhantes ao presente estudo, SILVA (2003) observou taxas de sobrevivência acima de 90\%, alimentando os camarões com ração especifica para engorda, o que pode ser mais um indicativo de que o alimento pode ter afetado a sobrevivência dos camarões no presente estudo. As taxas de sobrevivência dos

Ciência Rural, v.36, n.1, jan-fev, 2006. 


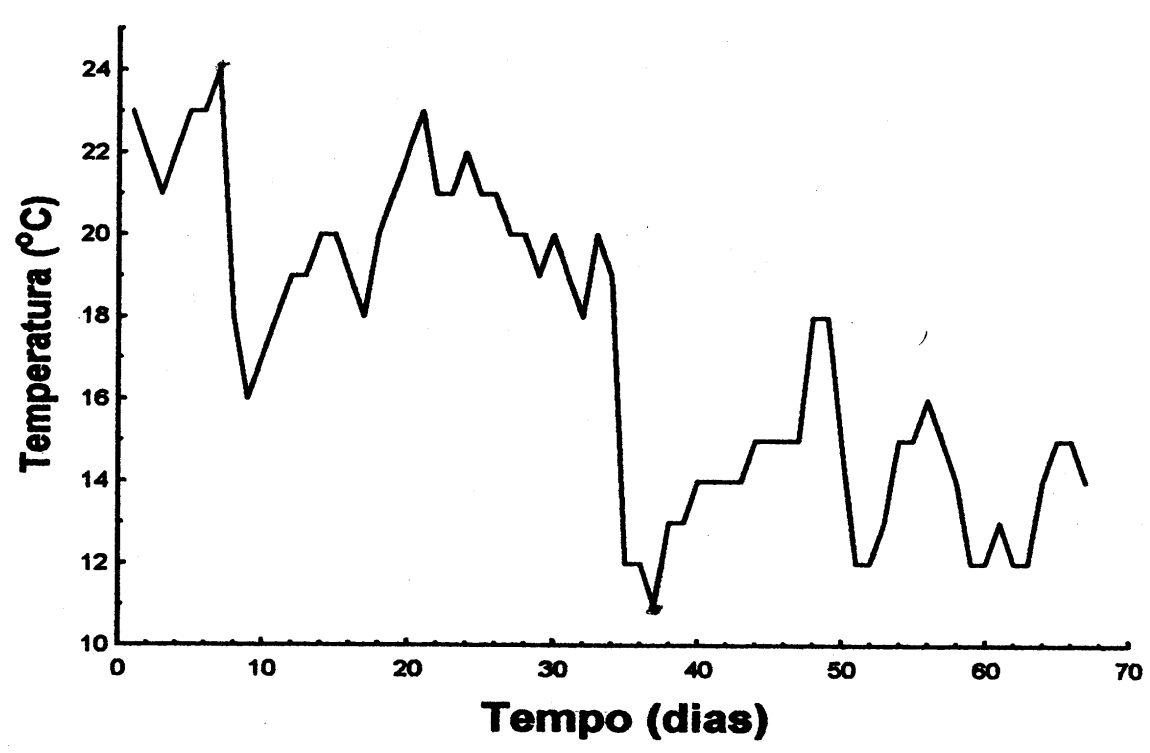

Figura 1 - Variação da temperatura da água ao longo do cultivo do camarão Farfantepenaeus paulensis em gaiolas durante o outono no estuário da Lagoa dos Patos, RS.

camarões mantidos em 20 e 40 camarões $/ \mathrm{m}^{2}$ podem ser consideradas satisfatórias. Sabe-se que em densidades elevadas, a sobrevivência dos camarões cultivados pode ser afetada, o que ocorre principalmente no momento da muda, quando os camarões estão mais suscetíveis ao canibalismo e às variações nas condições ambientais (WASIELESKY 2000). Assim como no presente trabalho, WASIELESKY et al. (2001) não encontraram diferenças significativas na sobrevivência de $\boldsymbol{F}$. paulensis nas densidades de 30 , 60,90 e $120 / \mathrm{m}^{2}$.

Os nossos resultados confirmam o efeito negativo da densidade de estocagem sobre o crescimento dos camarões cultivados em gaiola. SILVA (2003), cultivando F.paulensis em cercados nas densidades de 5, 10, 20 e 30 camarões $/ \mathrm{m}^{2}$, relatou pesos médios finais de 6,$3 ; 5,6 ; 4,7$ e 4,2g, respectivamente após 63 dias de cultivo. Este efeito negativo também

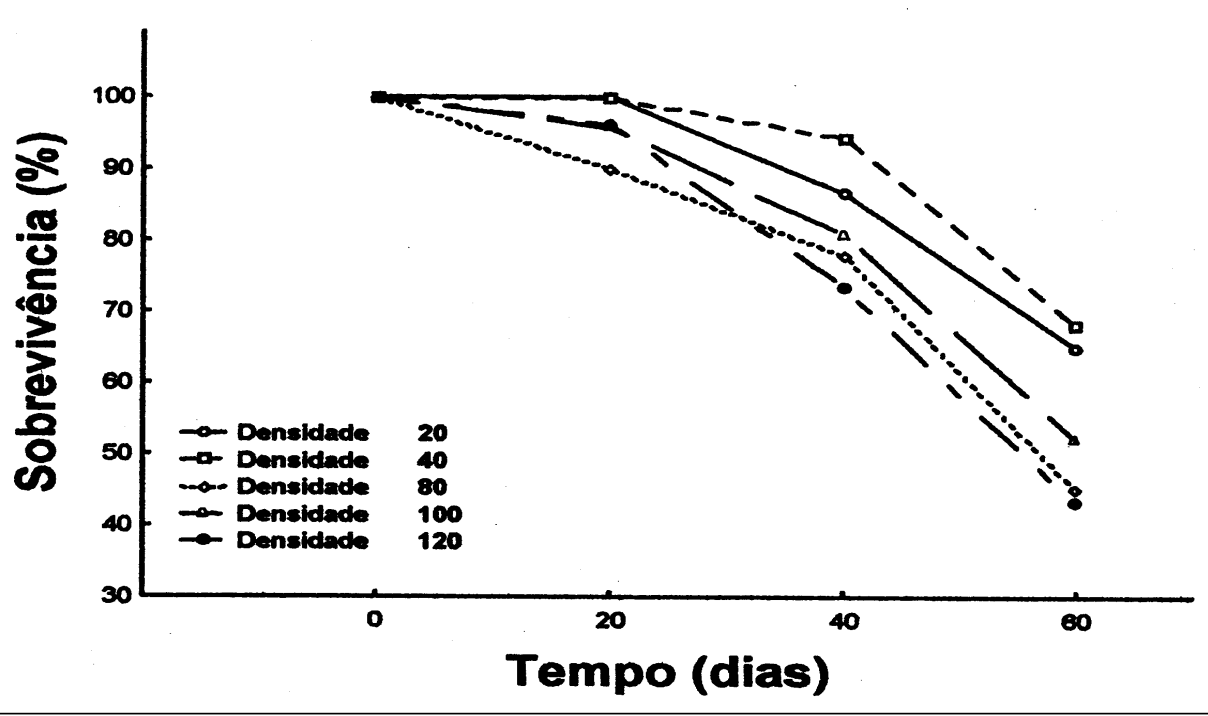

Figura 2 - Variação da sobrevivência ao longo dos 60 dias do cultivo do camarão Farfantepenaeus paulensis em gaiolas nas densidades de $20,40,80,100$ e $120 \mathrm{ind} / \mathrm{m}^{2}$

Ciência Rural, v.36, n.1, jan-fev, 2006. 


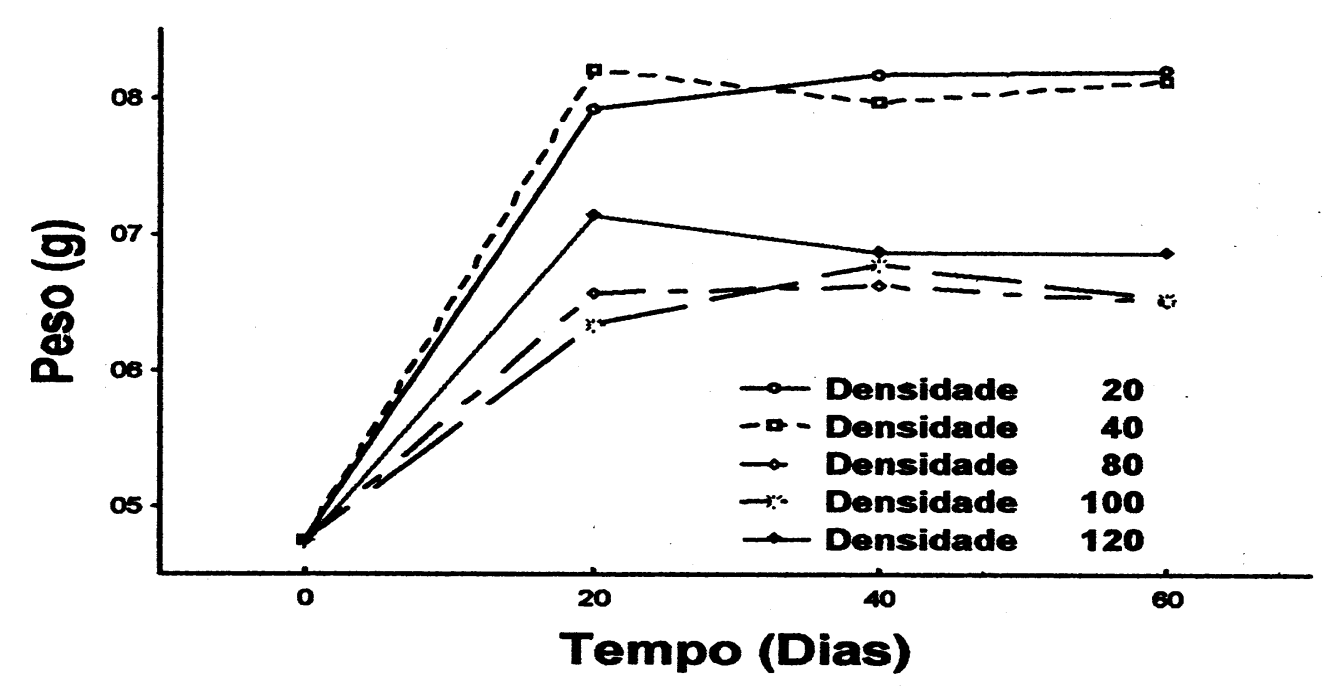

Figura 3 - Peso médio do camarão Farfantepenaeus paulensis cultivado em gaiolas sob diferentes densidades de estocagem nos 60 dias do experimento.

foi observado em outras espécies de peneídeos, como por exemplo Litopenaeus vannamei (SANDIFER et al. 1987) e Marsupenaeus japonicus (COMAN et al. 2004). Estes resultados, portanto, confirmam a relação inversa entre densidade de estocagem e crescimento de camarões peneídeos.

Em relação à biomassa final, a possibilidade de cultivar $\boldsymbol{F}$. paulensis durante o outono no sul do Brasil foi claramente demonstrada.Produziram-se entre 250 a $350 \mathrm{~g}$ de camarões com peso médio de $6,5-8,0 \mathrm{~g}$, mesmo em altas densidades de estocagem (40 a 120 camarões $/ \mathrm{m}^{2}$ ). Esses valores, comparados com o cultivo em viveiros, são equivalentes a uma produtividade de $2.500 \mathrm{~kg}$ a $3500 \mathrm{~kg}$ por hectare. Esses resultados confirmam os dados obtidos por outros autores em

Tabela 1 - Taxa de crescimento semanal (g/20dias), no período inicial (0-20 dias), intermediario (20-40 dias) e final (40-60 dias) do camarão-rosa Farfantepenaeus paulensis cultivado em diferentes densidades.

\begin{tabular}{llll}
\hline \multirow{2}{*}{$\begin{array}{l}\text { Densidade de } \\
\text { estocagem }\end{array}$} & \multicolumn{3}{c}{ Taxa de Crescimento Semanal } \\
\cline { 2 - 4 } & $0-20$ & $20-40$ & $40-60$ \\
\hline 20 & $1,10^{\mathrm{ab}} \pm 0,18$ & $0,09^{\mathrm{a}} \pm 0,06$ & $0,01^{\mathrm{a}} \pm 0,02$ \\
40 & $1,20^{\mathrm{a}} \pm 0,10$ & $0,02^{\mathrm{a}} \pm 0,04$ & $0,06^{\mathrm{a}} \pm 0,10$ \\
80 & $0,63^{\mathrm{bc}} \pm 0,07$ & $0,11^{\mathrm{a}} \pm 0,09$ & $0,06^{\mathrm{a}} \pm 1,10$ \\
100 & $0,54^{\mathrm{c}} \pm 0,37$ & $0,16^{\mathrm{a}} \pm 0,23$ & $0,00^{\mathrm{a}}$ \\
120 & $0,89^{\mathrm{bc}} \pm 0,07$ & $0,04^{\mathrm{a}} \pm 0,04$ & $0,02^{\mathrm{a}} \pm 0,02$ \\
\hline
\end{tabular}

Letras iguais numa mesma coluna indicam que não foram verificadas diferenças significativas $(p>0,05)$ entre densidades de estocagem. cultivos em gaiolas, os quais sugerem a utilização de densidades de estocagem superiores àquelas utilizadas nos cultivos convencionais em viveiros (WASIELESKY, 2000; SILVA, 2003).

Os resultados obtidos no presente trabalho demonstraram a possibilidade de cultivar $\boldsymbol{F}$. paulensis em gaiolas, durante o outono, no sul do Brasil, sendo recomendadas as densidades de estocagem de 40 a 120 juvenis $/ \mathrm{m}^{2}$, pois nas maiores a biomassa final foi maior. Contudo, quando a temperatura fica abaixo de $11^{\circ} \mathrm{C}$, o crescimento para e o cultivo em gaiolas passa a servir apenas como "manutenção" de camarões vivos para a venda na entra-safra. Sendo assim, essa é uma alternativa de renda para a comunidade de pescadores artesanais do município de Rio Grande e região.

\section{REFERÊNCIAS}

BALLESTER, E.L.C. et al. Influência do biofilme no crescimento do camarão-rosa Farfantepenaeus paulensis em sistemas de berçário. Atlântica, Rio Grande, RS, v.25, n.2, p.37-42, 2003.

COMAN, G. J. et al. The effects of density on the growth and survival of different families of juvenile Marsupenaeus japonicus Bate. Aquaculture, The Netherlands, v.229, p.215$223,2004$.

D'INCAO, F. Pesca e biologia de Penaeus paulensis na Lagoa dos Patos, RS. Atlântica, Rio Grande, RS, v.13, n.1, p.159169, 1991.

D'INCAO, F. et al. Avaliação da pesca de camarões nas regiões Sudeste e Sul do Brasil. Atlântica, Rio Grande, RS, v.24, p.103-116, 2002.

DOMINGOS, J.A. Cultivo do camarão-rosa Farfantepenaeus paulensis (Decapoda: Penaeidae) em gaiolas utilizando 
como alimentos ração comercial e resíduo de pescado preservados em sal. 2000. 34f. Monografia (graduação em Oceanologia) - Departamento de Oceanologia, Fundação Universidade Federal do Rio Grande.

JACKSON, C. J.; WANG, Y. G. Modelling growth rate of Penaeus monodon Fabricius in intensively managed ponds: effects of temperature, pond age and stocking density. Aquaculture Research, v.29 p.27-36, 1998

JENSEN, L. et al. Growth and survival of pink shrimp Farfantepenaeus paulensis postlarvae in cages and pen enclosures. Scientia Agricola, Piracicaba, v.61 p.332-335, 2003 .

KINNE, O. The efects of temperature and salinity on marine and brackish waters animal. II. salinity and temperature - salinity combinations. Oceanography and Marine Biology, New York, Annual review, p.281-339, 1964.

LIAO, I.C., CHIEN, Y.H. Culture of Kuruma prawn (Marsupenaeus japonicus Bate) in Asia. World Aquaculture, Louisiana, US, v.25 n.1, p.18-33. 1994. (Special Report).

LOMBARDI, J.V. et al. Floating cages in open sea water: an alternative for promoting integrated aquaculture in Brazil. Word Aquaculture Magazine Louisiana, USA, v.32, n.3 p.4750. 2001

LOWERY, R.S. Growth, molting and reproduction In : HOLDICH, D.M.; LOWERY, R.S. (Eds). Freshwater Crawfish: Biology management and explotation. Portland: Timber, 1988. p.83-113.

MUEDAS, W. et al. Manual de extensão sobre o cultivo de camarões marinhos. Florianópolis, SC: UFSC, 1997. 44p.

OLIVERA, A. et al. "Crescimento do Camarão-rosa" Penaeus paulensis no repovoamento da Lagoa de Ibiraquera, Santa Catarina, Brasil. In: SIMPÓSIO BRASILEIRO SOBRE CUltivo DE CAMARÃO, 4., 1993, João Pessoa. Anais... João Pessoa: MCR Aquacultura, 1993. V.1, p.439-451.

PAQUOTTE, P. et al. Intensive culture of shrimp Penaeus vannamei in floating cages: zootechnical, economic and environmental aspects. Aquacultute, Amsterdan, v.164, p.151-166, 1998.

PEIXOTO, S. et al. Comparative analysis of pink shrimp, Farfantepenaeus paulensis, and pacific white shrimp, Litopenaeus vannamei, culture in extreme southern Brazil. Journal of Applied Aquaculture, New York, v.14 p.101111,2003

SANDIFER, P.A. et al. Intensive culture potential of Penaeus vannamei. Journal World Aquaculture, v.18 n.2, p.94100,1987

SANTOS, M.H.S. Alimentação do camarão-rosa Farfantepenaeus paulensis (Pérez Farfante, 1967) (Decapoda, Penaeidae) cultivado. 2003. 229f. Tese (Doutorado em Oceanografia Biológica) - Departamento de Oceanologia, Fundação Universidade Federal do Rio Grande.

SILVA, O.A. Cultivo em cercados do camarão-rosa Farfantepenaeus paulensis (Pérez Farfante, 1967) em baixas densidades de estocagem. 2003. 34f. Monografia (graduação em Oceanologia) - Departamento de Oceanologia, Fundação Universidade Federal do Rio Grande.

SOARES, R.B. et al. Growth and food consuption of pink shrimp Farfantepenaeus paulensis postlarvae under different temperatures. Book of Abstracts. Aquaculture America, New Orleans, v1, p.307, 2000

WASIELESKY, W.J. Cultivo de juvenis do camarão-rosa Farfantepenaeus paulensis (Decapoda, Penaeidae) no estuário da Lagoa dos Patos: efeitos dos parâmetros ambientais. 2000. 199f. Tese ( Doutorado em Oceanografia Biológica) - Departamento de Oceanologia, Fundação Universidade Federal do Rio Grande.

WASIELESKY, W. J. et al. Effect of stocking density on pen reared pink shrimp Farfantepenaeus paulensis (Pérez-Farfante, 1967) (Decapoda, Penaeidae). Nauplius, v.9 n.2, p.163-167, 2001.

WYBAN, J.A.; SWEENEY, J.N. Intensive shrimp production technology: The Oceanic Institute Shrimp Manual. Honolulu: The Oceanic Institute, 1991. 158p. 